\title{
Drift Control of Pedestrian Dead Reckoning (PDR) for Long Period Navigation under Different Smartphone Poses ${ }^{\dagger}$
}

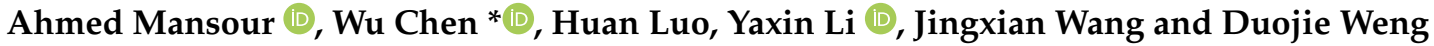 \\ Department of Land Surveying and Geo-Informatics, The Hong Kong Polytechnic University, Hong Kong; \\ ahmed.m.mostafa@connect.polyu.hk (A.M.); hilary.luo@connect.polyu.hk (H.L.); \\ yaxin.pu.li@connect.polyu.hk (Y.L.); jingxian.wang@connect.polyu.hk (J.W.); duojieweng@gmail.com (D.W.) \\ * Correspondence: wu.chen@polyu.edu.hk \\ + Presented at 8th International Electronic Conference on Sensors and Applications, 1-15 November 2021; \\ Available online: https://ecsa-8.sciforum.net.
}

Citation: Mansour, A.; Chen, W.; Luo, H.; Li, Y.; Wang, J.; Weng, D. Drift Control of Pedestrian Dead Reckoning (PDR) for Long Period Navigation under Different Smartphone Poses. Eng. Proc. 2021, 10, 21. https://doi.org/10.3390/ ecsa-8-11302

Academic Editor: Stefano Mariani

Published: 1 November 2021

Publisher's Note: MDPI stays neutral with regard to jurisdictional claims in published maps and institutional affiliations.

Copyright: (c) 2021 by the authors. Licensee MDPI, Basel, Switzerland. This article is an open access article distributed under the terms and conditions of the Creative Commons Attribution (CC BY) license (https:/ / creativecommons.org/licenses/by/ $4.0 /)$.

\begin{abstract}
The inherent errors of low-cost inertial sensors cause significant heading drift that accumulates over time, making it difficult to rely on Pedestrian Dead Reckoning (PDR) for navigation over a long period. Moreover, the flexible portability of the smartphone poses a challenge to PDR, especially for heading determination. In this work, we aimed to control the PDR drift under the conditions of the unconstrained smartphone to eventually enhance the PDR performance. To this end, we developed a robust step detection algorithm that efficiently captures the peak and valley events of the triggered steps regardless of the device's pose. The correlation between these events was then leveraged as distinct features to improve smartphone pose detection. The proposed PDR system was then designed to select the step length and heading estimation approach based on a real-time walking pattern and pose discrimination algorithm. We also leveraged quasi-static magnetic field measurements that have less disturbance for estimating reliable compass heading and calibrating the gyro heading. Additionally, we also calibrated the step length and heading when a straight walking pattern is observed between two base nodes. Our results showed improved device pose recognition accuracy. Furthermore, robust and accurate results were achieved for step length, heading and position during long-term navigation under unconstrained smartphone conditions.
\end{abstract}

Keywords: indoor positioning; PDR; inertial sensors; heading drift; unconstrained smartphone

\section{Introduction}

Location awareness facilitates our daily lives significantly by involving in a wide range of services, including commercial location-based services (LBSs) and life-saving services [1]. Outdoors, Global Navigation Satellite Systems (GNSSs) provide a satisfactory performance that meets most LBS requirements. Indoors, however, GNSS performance degrades significantly due to signal blockage and attenuation. Many Indoor Localization Systems (ILSs) have been proposed to fulfill the LBS needs. Portable smartphones are the most common platforms for existing ILSs [2], thanks to the enormous capabilities of the embedded sensors and the widespread use of these devices. The Pedestrian Dead Reckoning (PDR), based on gait cycle analysis, has been intensively studied in the last two decades. PDR has gained great popularity as a low-cost and autonomous technique that relied only on off-the-shelf inertial sensors [1]. A typical PDR scheme consists of three main components: step detection, step length and heading estimation. PDR provides a reliable short-term solution that can bridge outages of wireless techniques and establish the system's model in hybrid systems. However, when PDR has been used as a standalone technique for long-term navigation, its performance degrades rapidly over time due to the accumulated drift of the gyro heading [1]. In addition, the smartphone flexible portability poses a challenge for PDR, owing to the difficulty of predicting the effect of an unconstrained smartphone on the PDR solution [3]. 
In the literature, numerous research efforts have been devoted to enhancing the heading estimation $[2,4,5]$. Regarding gyro heading drift, since this drift cannot be measured without an external calibration source [1], magnetometer angular rate gravity sensors (MARG) have been proposed to calibrate this drift [4]. However, magnetic fields (MF) can be easily disturbed indoors, leading to severe heading errors. Quasi-static magnetic field (QSMF) [2] and joint QSMF [5] methods have been devised to capture reliable compass heading and overcome the high perturbation of MF. However, it is difficult to frequently capture such QSMF measurements in complex indoor environments filled with electrical and metallic components. Regarding the unconstrained smartphone conditions, several studies have been conducted to distinguish the device pose [3,6]. Wang et al. [6] extracted time and frequency domain features from a sliding window of the user acceleration and angular velocities to distinguish the device pose. In [3], accumulated absolute acceleration and angle change of roll, pitch and yaw were utilized for pose detection. To assess the performance of step detection under unconstrained smartphones, thorough comparisons among different step count approaches have been conducted in [7]. The assessment concluded that the time-based peak detection method is the optimal detection technique regardless of the device pose. Deng et al. [8] proposed a robust heading estimation for inpocket pose using the Principal Component Analysis (PCA) method. Zheng et al. [9] used the rotation vector sensor to improve the swinging pocket and mode heading estimation.

Despite plentiful research studies, the heading drift and the effects of the unconstrained smartphone remain open problems that limit the use of PDR in long-term navigation. In this paper, our overarching aim is to control the PDR drift under the conditions of the unconstrained smartphone to eventually enhance the PDR performance. To this end, we developed a robust step detection mechanism that efficiently captured the peak and valley events of the triggered steps, regardless of the device pose. The correlation between these events was then leveraged as distinct features to improve smartphone pose detection. The proposed PDR system was then designed to select the step length and heading estimation approach based on the feedback from a real-time walking pattern and pose discrimination algorithm. Additionally, we leveraged QSMF measurements that have less MF disturbance to estimate reliable absolute heading. We also calibrated the step length and heading when a straight walking pattern was observed between two base nodes (i.e., emitters with predefined location, e.g., BLE beacons or GNSS observations with accurate solution). Specifically, our work makes the following contributions:

1. We adopt the pose detection method proposed in [6] by combining the relationship between the successive peaks and valleys of the triggered steps as a distinct feature for better detection of the smartphone poses.

2. We mitigated the gyro heading drift employing two calibration methods (i.e., QSMF and the straight walking between two base nodes).

The remainder of this article is organized as follows: Section 2 discusses the proposed method. The experimental work and results are presented in Section 3. In the last section, the conclusion is summarized.

\section{Methods}

Figure 1 shows the framework of the proposed PDR scheme. The methodology section is organized as follows: the step detection mechanism is first illustrated since we depend on its results (i.e., the steps peak and valley acceleration values) for the pose detection that started after that. The step length and heading estimation approaches are then explained. Finally, the position estimation and outlier filtering are discussed. 


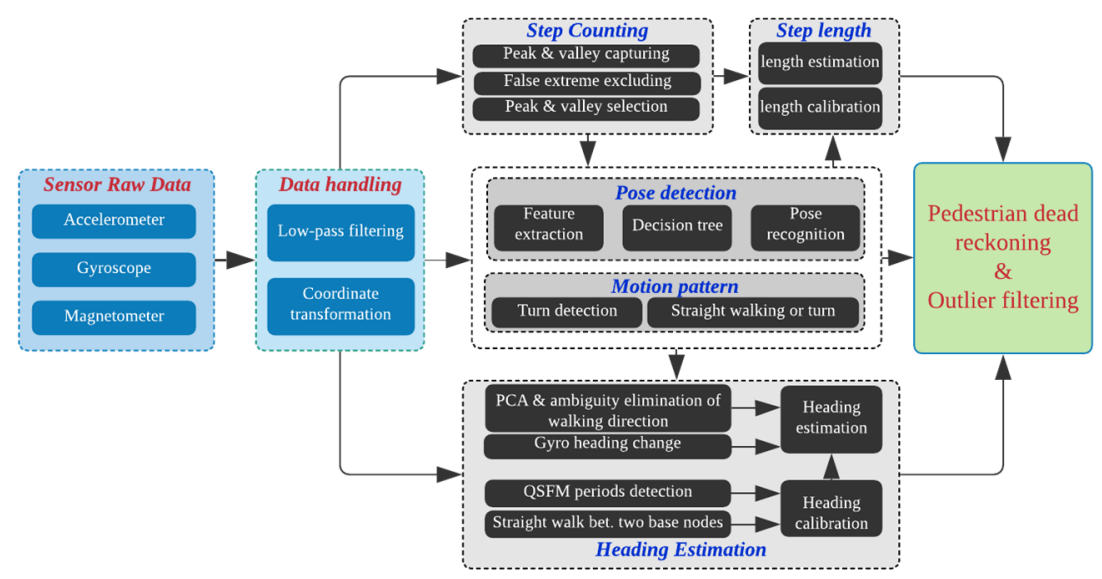

Figure 1. The architecture of the proposed PDR system.

\subsection{Step Detection}

The time-based peak and valley detection approach was selected to detect the triggered steps regardless of the smartphone pose, as recommended in reference [7]. We handled the raw acceleration for the detection task by conducting the following four steps:

1. The effect of Earth's gravity was eliminated using a low-pass filter to obtain the real linear acceleration applying on the device.

2. The optimal axis that provides a strong indication of the step signatures, regardless of the device's pose, was obtained (i.e., the vertical axis relative to Earth's surface). The acceleration applying on this axis was estimated by converting the linear acceleration from the Device Coordinate System to the Navigation Coordinate System.

3. The random noise effect was alleviated using a low-pass filter.

4. The peak and valley acceleration for each step was captured based on the gait cycle characteristics. To ensure excluding the slight hand trembling from being counted as a step, two thresholds were imposed on the duration and extreme values as follows:

$$
\left\{\begin{array}{l}
\delta T_{\text {step }} \geq \delta T_{\text {th }} \\
A_{\text {peak }} \geq A_{\text {th }}, A_{\text {valley }} \geq A_{\text {th }}
\end{array}\right.
$$

where $\delta T_{\text {step }}$ and $\delta T_{\text {th }}$ are the time duration between two adjacent peaks and their threshold, respectively. $A_{\text {peak }}, A_{\text {valley }}$ and $A_{\text {th }}$ are the peak, valley acceleration of the detected step and their threshold, respectively.

\subsection{Walking Pattern and Pose Detection}

Both the walking pattern (i.e., including straight walking and making a real turn) and device poses (i.e., including the handheld, calling, swinging and in-pocket poses) were detected. To recognize the user walking pattern, a real-time turn detection algorithm was developed based on the maximum difference of the gyro heading change of a sliding window. Two levels of heading change (i.e., $15^{\circ}$ and $40^{\circ}$ ) were used to determine the start and end of the real turn. Capturing the turn events precisely enabled us to distinguish the straight walking pattern and help to control the gyro heading drift while walking straight.

The main concept behind distinguishing the device poses stems from exploiting their distinct acceleration and device angular change characteristics. The filtered data were inadequate to recognize the device pose correctly. Thus, features were extracted from the handled acceleration and angular change recorded through a sliding window to augment the detection decision. Based on analysis of the device's poses' features conducted in [6], the following features were extracted from each window:

1. The acceleration variances in $\mathrm{x}, \mathrm{y}, \mathrm{z}$ directions and magnitude $\left(\sigma_{a x}^{2}, \sigma_{a y}^{2}, \sigma_{a z}^{2}, \sigma_{a}^{2}\right)$.

2. The angular velocities variances in $\mathrm{x}, \mathrm{y}, \mathrm{z}$ directions and magnitude $\left(\sigma_{\omega x}^{2}, \sigma_{\omega y}^{2}, \sigma_{\omega z}^{2}, \sigma_{\omega}^{2}\right)$. 
3. The energy spectral density of $x, y$ angular velocity and acceleration $\left(S_{\omega x}, S_{\omega z}, S_{a}\right)$.

In addition, we boosted the abovementioned features by the acceleration differences between the successive peaks and valleys ( $A_{\text {peak }}^{k}, A_{\text {valley }}^{k}$ ), where $k$ is the current step since the differences between the successive extremes have a distinct range in each pose. Finally, a decision tree was utilized to decide the device pose, as can be seen in Figure 2. $\tau$ terms are the predefined thresholds for the selected features. Evidence of the difference range of each device pose is discussed in Section 3, Figure 3.

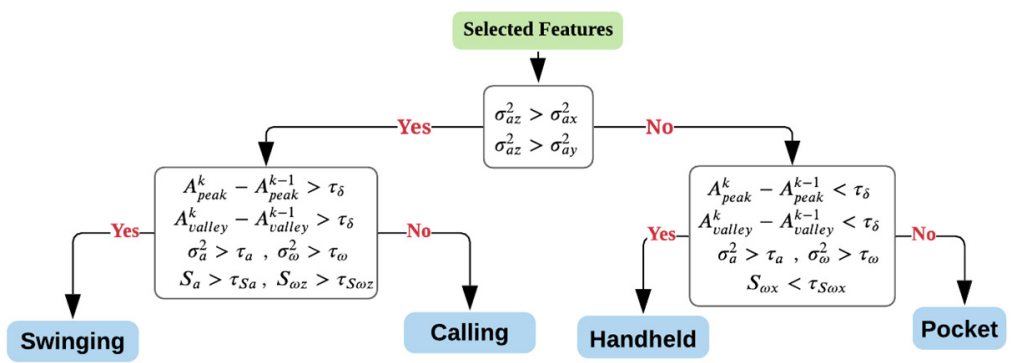

Figure 2. Device pose detection decision tree.
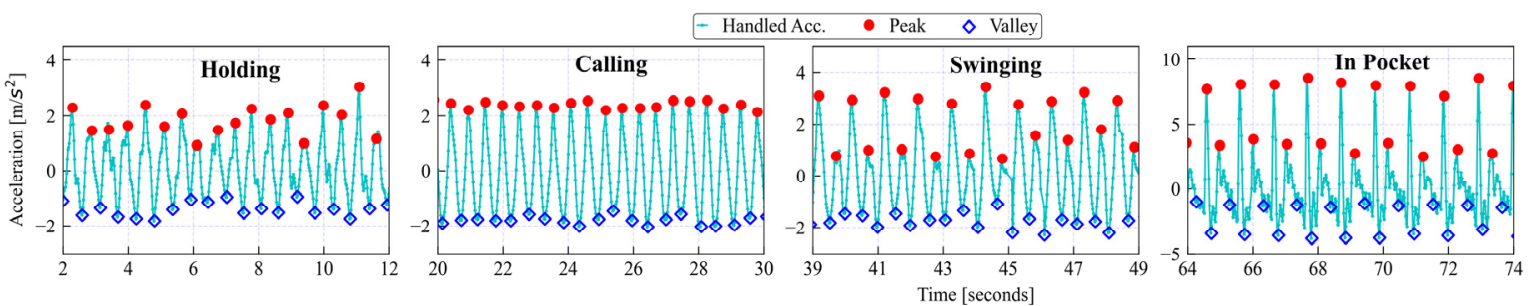

Figure 3. Step detection performance under different smartphone poses.

\subsection{Step Length Estimation}

The step length was dynamically estimated based on a nonlinear model introduced in [10], which supposed that the length is nonlinearly correlated with the total vertical acceleration change of each step as the following formula:

$$
L_{k}=c .\left(A_{\text {peak }}-\left|A_{\text {valley }}\right|\right)^{0.25} 0.6 \leq L_{k} \leq 0.9
$$

where $A_{\text {peak }}$ and $A_{\text {valley }}$ are the peak and valley acceleration for step $k$, respectively. $c$ is the pedestrian's profile parameter. This model provided reliable step length for specific walking situations (i.e., when the user carries a smartphone steadily in handheld or calling pose). In-pocket, swinging hand poses, the total change in vertical acceleration within one step would be prone to sharp fluctuations that do not reflect the step length and lead to false estimation of the user displacement. In real daily life scenarios, to continuously estimate a reliable step length, we considered the following circumstances:

1. The false peaks were filtered out to avoid false step length estimation.

2. When the handheld and calling poses were detected, the nonlinear model was applied and the empirical step length was computed and recorded, while in the case of a swinging hand or in-pocket, the stored mean step length was used.

3. $\quad c$ was calibrated when a straight walking pattern was observed between two base nodes with a steady handheld or calling pose.

\subsection{Step Heading Estimation}

The attitude of the device relative to the user body greatly affects the heading estimation. In handheld and calling poses, the attitude is relatively stable. Thus, the heading offset between the walking direction and the smartphone's orientation can be simply esti- 
mated. In contrast, in swinging and pocket poses, the orientation of the smartphone and the offset varies constantly. Based on the high variation of the horizontal acceleration being parallel to the moving direction, the PCA-based approach [8] was leveraged to compute local headings by estimating the first eigenvector. Since the estimated direction from PCA fails to distinguish whether the direction is $\varphi$ or $\varphi+180$, we selected the closest angle to the compass heading to solve this ambiguity. Owing to the low credibility of the compass heading in the short-term solution, we estimated a mean value in a sliding window to improve the heading robustness. The gyro's angular velocities were used to estimate the relative heading change through numerical integration. A weighted heading fusion was used to combine the absolute compass heading and gyro relative heading based on the correlation between them and the stability of the compass heading when QSMF is detected. Furthermore, we calibrated the estimated heading when a straight walking pattern was observed between two base nodes thanks to the ability to detect the turns.

\subsection{Position Estimation and Outlier Filtering}

The user position was calculated based on the previous position, step length and heading [6]. To curb the position estimation from the drift that stems from the slight body shaking, we developed a real-time outlier filtering using the turn detection algorithm. When no turn was detected, the random sample consensus method [11] was applied to construct the optimal parameter of the linear model that fit the walked steps.

\section{Experiments and Results}

Real experiments were conducted on floor six of Block $Z$ at the Hong Kong Polytechnic University, using Huawei Mate 20 Pro smartphone. A $50 \mathrm{~Hz}$ sampling frequency was used for both accelerometers and magnetometers. A higher sampling frequency $100 \mathrm{~Hz}$ was used for the gyro to estimate accurate heading changes.

To evaluate the PDR performance, each PDR element was tested separately. Regarding the detection of the triggered steps, tests were conducted on a long corridor using four smartphone poses (handheld, calling, swinging and in-pocket). The results for the detection test considering the handheld pose showed mean and maximum detection error of about $0.6 \%$ and $2 \%$, respectively. In the calling pose, better detection results were achieved with a maximum error of around $1.5 \%$. The swinging pose had a mean error of $2.4 \%$. A fluctuated performance was observed with in-pocket pose with a higher number of false extremes, with a maximum error of 3.3\%. Figure 3 shows the step detection results with different poses. The discrimination accuracy of the device pose was about $96.8 \%, 98.2 \%, 97.4 \%$ and $96.4 \%$ using the peak and valley features compared to $96.2 \%, 93.8 \%, 97.1 \%$ and $95.1 \%$ obtained in [6] for holding, calling, swinging and in-pocket poses, respectively.

A closed rectangular track (see Figure 4) was selected to assess the step length estimation. The walked distance was computed as the sum lengths of the triggered steps. The results showed that the step length estimation under device poses recognition obtained a maximum error of $1.8 \mathrm{~m}$ with a standard deviation less than $0.9 \mathrm{~m}$ for about $75 \mathrm{~m}$ total distance.

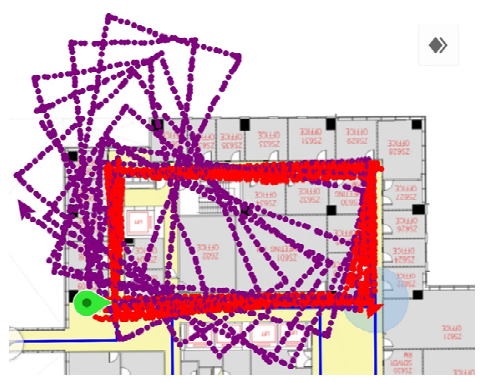

Figure 4. Long-term PDR performance. 
The same track was also repeated ten times to evaluate the long-term heading estimation performance. The compass heading had a sharp variation in short-term solution with maximum error reaching $50^{\circ}$. The cumulative gyro heading error approached $90^{\circ}$ after $20 \mathrm{~min}$ of walking. The proposed heading fusion scheme provided robust long-term heading performance with maximum drift less than $4^{\circ}$. The walking direction was obtained with drift less than $10^{\circ}$ in swinging and pocket poses. This drift was calibrated when QSMF periods or walking straight between two base nodes were detected. Figure 4 shows a comparison between the positioning performance of the proposed PDR and the PDR-based gyro heading. The PDR-based gyro heading (the purple track, Figure 4) for loops one and two (i.e., short-term solution) showed similar performance to the proposed PDR (the red track, Figure 4). However, the purple track was rotated around $100^{\circ}$ after walking $750 \mathrm{~m}$. This was due to the accumulated heading drift with the long-term localization. In contrast, the red track achieved an accurate positioning solution over time with a mean and a $90 \%$ error of 1.45 and $2.35 \mathrm{~m}$, respectively.

\section{Conclusions}

In this paper, we proposed a PDR scheme to curb the PDR drift under unconstrained smartphone conditions. A robust step detection was developed based on time-domain peak and valley acceleration extremes. The differences between the successive extremes were used to boost the detection decision of the device pose. The proposed PDR scheme was devised to select the step length and direction approach based on the current device pose. The QSMF data were applied to calibrate the gyro heading. Moreover, the step length and heading were also compensated when a straight walking pattern was observed between two base nodes. We achieved reliable step detection results under different smartphone poses. The pose recognition was enhanced and helped improve the step heading and length estimation. Furthermore, the estimated heading after $20 \mathrm{~min}$ of walking drifted less than $5^{\circ}$. The overall performance of PDR was significantly improved in long-term scenarios with a mean and a $90 \%$ error of 1.45 and $2.35 \mathrm{~m}$, respectively. Overall, the proposed PDR scheme was examined and performed well in common confined indoor spaces such as corridors. However, the scheme could be further investigated in open indoor spaces. The proposed PDR scheme can be further integrated with various wireless location systems, such as WiFi and Bluetooth technologies based on fingerprinting methods. Future studies could examine utilizing pervasive base nodes in the buildings to achieve a ubiquitous system.

Author Contributions: Conceptualization, A.M. and W.C.; software, A.M. and Y.L.; validation, H.L. and J.W.; writing—original draft preparation, A.M., writing—review and editing, A.M. and D.W.; supervision, W.C. All authors have read and agreed to the published version of the manuscript.

Conflicts of Interest: The authors declare no conflict of interest.

\section{References}

1. Hou, X.; Bergmann, J. Pedestrian dead reckoning with wearable sensors: A systematic review. IEEE Sens. J. 2021, 21, 143-152. [CrossRef]

2. Li, Y.; Georgy, J.; Niu, X.; Li, Q.; El-Sheimy, N. Autonomous calibration of MEMS gyros in consumer portable devices. IEEE Sens. J. 2015, 15, 4062-4072. [CrossRef]

3. Deng, Z.; Liu, X.; Qu, Z.; Hou, C.; Si, W. Robust heading estimation for indoor pedestrian navigation using unconstrained smartphones. Wirel. Commun. Mob. Comput. 2018, 2018, 5607036. [CrossRef]

4. $\mathrm{Hu}, \mathrm{J} . ; \mathrm{Sun}, \mathrm{K}$. A Robust orientation estimation algorithm using MARG sensors. IEEE Trans. Instrum. Meas. 2015, 64, 815-822. [CrossRef]

5. Yan, D.; Shi, C.; Li, T. An improved PDR system with accurate heading and step length estimation using handheld smartphone. J. Navig. 2021, 74, 1-19. [CrossRef]

6. Wang, B.; Liu, X.; Yu, B.; Jia, R.; Gan, X. Pedestrian dead reckoning based on motion mode recognition using a smartphone. Sensors 2018, 18, 1811. [CrossRef] [PubMed]

7. Brajdic, A.; Harle, R. Walk detection and step counting on unconstrained smartphones. In Proceedings of the 2013 ACM International Joint Conference on Pervasive and Ubiquitous Computing, Zurich, Switzerland, 8-12 September 2013; pp. 225-234.

8. Deng, Z.-A.; Wang, G.; Hu, Y.; Wu, D. Heading estimation for indoor pedestrian navigation using a smartphone in the pocket. Sensors 2015, 15, 21518-21536. [CrossRef] [PubMed] 
9. Zheng, L.X.; Zhan, X.Q.; Zhang, X.; Wang, S.Z.; Yuan, W.H. Heading estimation for multimode pedestrian dead reckoning. IEEE Sens. J. 2020, 20, 8731-8739. [CrossRef]

10. Weinberg, H. Using the ADXL202 in pedometer and personal navigation applications. Analog. Devices AN-602 Appl. Note 2002, 2, 1-6.

11. Ahmed, W.; Shi, W.; Wenbin, X. Modeling complex building structure (LoD2) using image-based point cloud. In Proceedings of the 2018 IEEE International Conference on Image Processing, Applications and Systems (IPAS), Sophia Antipolis, France, 12-14 December 2018; pp. 110-114. 